\title{
QSAED3, a Program for Quantification and Processing of SAED Patterns
}

\author{
X.-Z. $\mathrm{Li}^{1}$
}

${ }^{1 .}$ Nebraska Center for Materials and Nanoscience, University of Nebraska, Lincoln, NE.

Selected-area electron diffraction (SAED) analysis has been extensively used in materials science for phase identification, interpretation of twins and coexisted multiple phases and so forth. SAED patterns from a single grain and from polycrystalline area in TEM samples are common in essence but different in many aspects, so we treat the two cases separately for quantification and processing of SAED patterns. QPCED has been developed for quantification and processing of polycrystalline SAED patterns [1,2] and QSAED has been developed for single crystalline SAED patterns [3].

The current version QSAED3 has much improvement in the design and the functionality over the previous version [3]. The software design has features, such as (i) a frame with a drop-down menu, a graphical menu bar and a display panel; (ii) the processing operation and parameters input are carried out in corresponding dialog windows; (iii) a drag-drop-box or a regular window file system for loading input SAED patterns; (iv) both of the processed data and displayed images can be saved to files; (v) output image can be defined its region of interest and its resolution. Figure 1 shows (a) the main interface of QSAED3 with retrieved intensities of an experimental SAED pattern, (b) a tool for magnifying a local area of the experimental SAED pattern (c) a background curve.

The main application of QSAED3 is to retrieve/display the diffraction intensities and lattice spacings of SAED patterns, including precession electron diffraction (PED) patterns. A 1-D intensity profile along a line and a 2-D distribution of intensities can be generated after subtracting the background intensities. The basic reciprocal lattice spacing can be precisely measured to sub-pixel and converted to nm using the scaling bar. The intensities of reflections in the pattern may be adjusted according to its crystallographic symmetry as shown in Figure 2. QSAED3 also provides image processing functions, e.g. resizing, rotating, inverted contrast and cutting into a square, which make it easy to prepare the experimental SAED patterns for publication.

QSAED3 was written in Java SE8. Further code optimization (including obfuscation) was carried out for the compiled class files. Java Runtime Environment (JRE) and Java Advanced Imaging (JAI) are required to run QSAED3. The executable code, user manual, specification and a set of crystal structural data are available at http://www.unl.edu/ncmn-cfem/xzli/computer-programs .

The installation and usage of QSAED3 is easy and straightforward, (i) download and install the most recent JRE and JAI from Java official site; (ii) download and decompress the QSAED3.7z or LANDYNE3.7z; (iii) copy the license file to the same fold with the program (qsaed3.exe or landyne3.exe); (iv) double click the icon of QSAED3.exe or click the QSAED3 in Landyne launcher to start the software. A step-by-step guide is listed in the user manual. QSAED3 is one of the components in LANDYNE software suite, it will be more powerful to use the components together in the integrated package. The purpose of the LANDYNE software suite is twofold: i) as research tools to analyze experimental results, ii) as teaching tools to show students the principles of electron diffraction and crystallography. 
References:

[1] X-Z Li, Microsc. Microanal. 17(S2) (2011), p. 1096.

[2] X-Z Li, J. Appl. Cryst. 45 (2012), p. 862.

[3] X-Z Li, Microsc. Microanal. 20(S3) (2014), p. 1486.

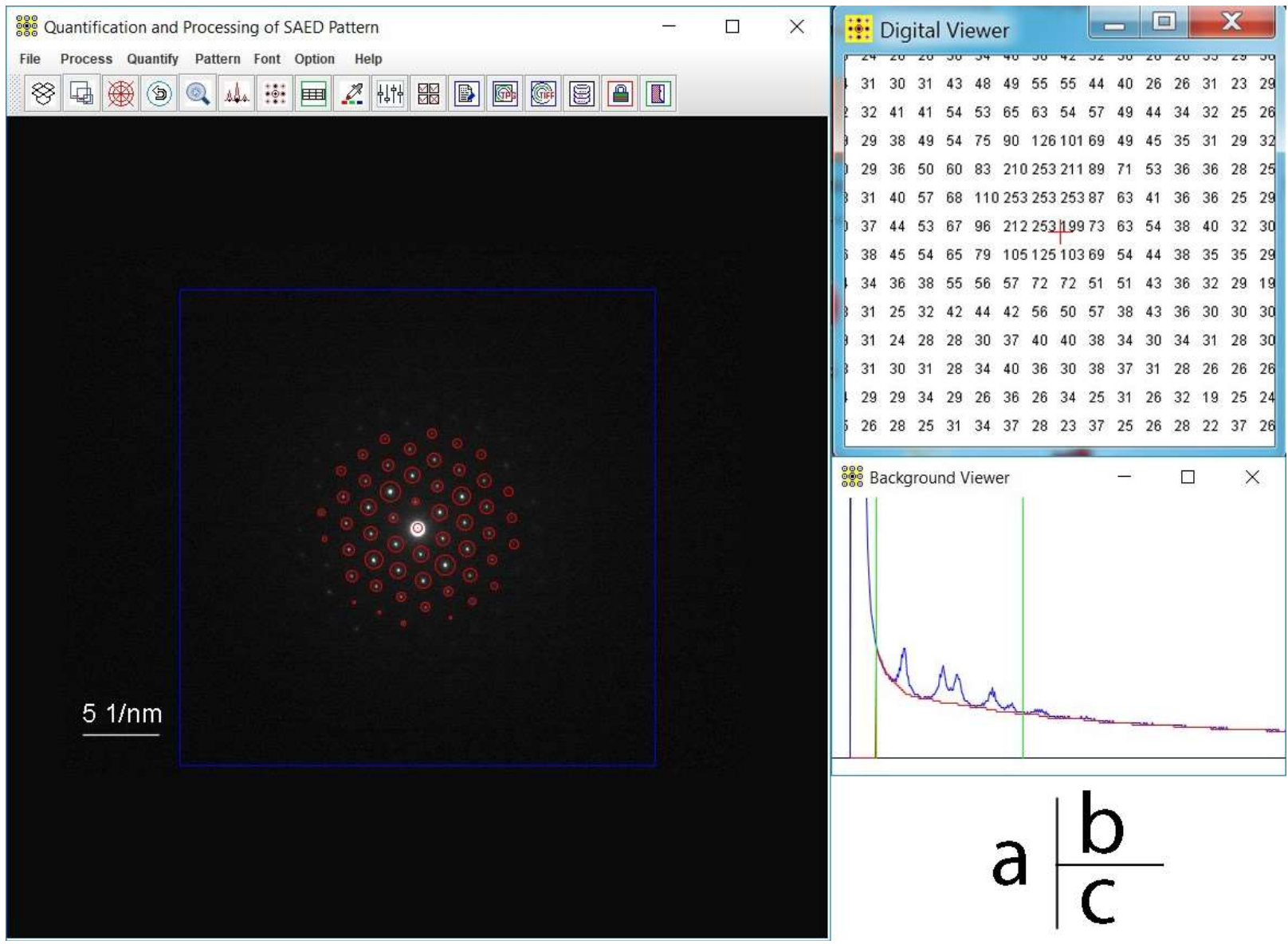

Figure 1. (a) Main GUI of QSAED3 with retrieved intensities of an experimental SAED pattern, (b) a tool for magnifying a local area of the experimental SAED pattern (c) a background curve.
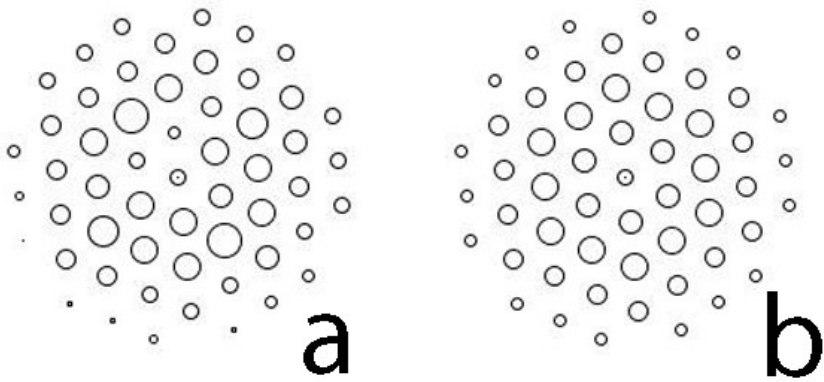

Figure 2. (a) Retrieved intensities of the experimental SAED pattern in Figure 1 and (b) a processed the pattern according to its crystallographic symmetry. 